\title{
CYTOCHEMICAL AND IMMUNOCHEMICAL ANALYSIS AT THE ELECTRON MICROSCOPY LEVEL: OBTAINING CONTRASTING ANTIBODIES BY USE OF IODINE
}

\author{
By L. B. MEKLER, S. M. KLIMENKO, G. E. DOBREZOV, V. K. NAUMOVA, \\ YU. P. HOFFMAN and Prof. V. M. ZHDANOV \\ D. I. Ivanovsky Institute of Virology, Academy of Medical Sciences of the U.S.S.R., Moscow
}

$\mathrm{M}$ ODERN histochemistry offers various methods of histochemical analysis ${ }^{1}$. These methods have been developed on the basis of optic microscopy. However, limitations due to the low-resolving capacity of optical microscopes do not allow the use of these methods for investigating histochemical problems at the molecular level.

With the use of electron microscopes the only way of revealing two different neighbouring structures or substances is by contrasting. The intensity of blackening of a photographic plate (other conditions being equal) is determined by the quantity of electrons reaching each particular area of the plate. The quantity of electrons loosened by the original electron pencil passing through the object is detormined by that part of the electron pencil which is scattered inelastically and elastically by the atoms of the object. The first effect is determined by the correla. tion between the energy of the falling electrons and the structure of electron levels of an atom. It occurs if the energy of the falling electrons is equal to or more than the energy which corresponds to characteristic frequencies of the element spectrum.

If interaction between the electron pencil and the atoms of the element is inelastic, the representation of the biological object in principle can be obtained as a mosaic which reflects the distribution of the chemical element in the preparation. In this case the change of the electron energy (for example, by a change of the accelerating voltage) and construction of an image of the object examined by inelastically scattered electrons renders it possible to obtain some photographs which reflect the distribution of different elements in the object investigated.

If one proceeds from the $K$-lines of the X-ray spectrum the excitation energy of which is closest to the energy of electrons now used in electron microseopy, a simple calculation shows that micro-elements most interesting from the biological point of view (manganese, iron, cobalt, zinc, molybdenum, silver, iodine, mercury, lead, etc.) may be detected with an electron microscope operating at 7-100 $\mathrm{kV}$ with sufficiently precise stabilization of the voltage (the error should not exceed 0.001 per cent), a high resolving power $(\sim 2 \AA)$ and corresponding system of construction of image. At present such microscopes do not exist. However, possibilities of the method already mentioned may make the designing of such electronic microscopes very desirable.

The second effect, that is, elastic scattering, is determined in common by the size of the atom. Tho higher the atomic numbor of the element, the more intense (other conditions being equal) is the scattering of the electrons by its atoms.

This effect apparently cannot bo used for identification of elements, because the additivity of scattering effect on many atoms does not allow estimation of the part brought in common scattering by overy element.

At present, electron microscopy does not render it possible to distinguish between elastic and inelastic scattering. Both effects are determined by the size of atomic cross-section for electron scattering. If the atomic number is increased the cross-section for electron scattering is also increased. Owing to this it is easy to distinguish two structures containing atoms with widely distant atomic numbers, but it is difficult to do this when the atomic numbers are neighbouring. For this purpose the heavy elements (osmium, manganese, iron, uranium) are used as specific markers for increasing the contrast of objects. The specificity of a marker is highly increased by combining the iron-contained protein, ferritin, with antibodies. The possibility of obtaining antibodies against 'artificial' antigens ${ }^{3,4}$ suggests the idea of developing a method of electron-microscopic immunochemical analysis which is highly sensitive and unique as a specific immunochemical method, on one hand, and render it possible to detect both the macromolecules and usual smaller molecules of organic substances on the other. Hence, it is evident that the method of obtaining contrasting antibodies is of great importance.

However, the method of ferritine antibodies has two significant disadvantages; for example: (1) obtaining a sufficiently pure complex molecule of antibody-ferritine is very difficult and laborious; (2) a sharp increase in the size of the immunologically active molecule may impede its diffusion to the areas of antigen localization in the cell.

To eliminate these disadvantages, attempts were made to develop a method based on combining the antibody molecule with a small organic molecule containing mercury $^{5,6}$. This method requires a scarcely available mercurycontaining organic compound and is also very laborious.

It is well known, however, that up to 20 iodine atoms may be included in the antibody molecule without losing its specific activity ${ }^{7,8}$. The problem is whether this amount of iodine is sufficient to make the antibody thus iodized 'black' enough, that is, contrasting in the field of an electronic microscope against a background of the neighbouring areas of the preparation.

At present there are no theoretical data allowing one to calculate precisely the dependence between the quantity of atoms with a particular atomic numbor and the level of scattering of electrons produced by them, that is, the value of blackening observed and the contrast in the electron microscopy. On the basis of the theoretical approach $^{2}$, Valentine ${ }^{10}$ developod an equation making it possible to calculate as a guide the number $(N)$ of atoms with the atomic number $(\boldsymbol{Z})$ which must be distributed uniformly over the surface of an object or in its volume to obtain a minimally detectable increase in the contrast at the particular voltage used for electron-microseopic examination.

By using this equation, Pearse ${ }^{1}$ carried out a similar calculation for contrasting with $\mathrm{OsO}_{4}$. According to his results sufficient contrast may be obtained by introducing one residue of $\mathrm{OsO}_{2}$ per $400 \AA$ (ref. 2) of the membrane surface. Since the cross-section of the antibody is $8000 \AA$ (ref. 2) and the atomic number of iodine $(Z)$ is 53 , it is evident that for obtaining a contrast analogous to that produced by osmium one should introduce about 40 iodine atoms into the antibody molecule.

An important factor for obtaining the contrast is the distribution of iodine atoms in the antibody molecule, determined in its turn by the distribution of tyrosine residues. Unfortunately such data are lacking. 


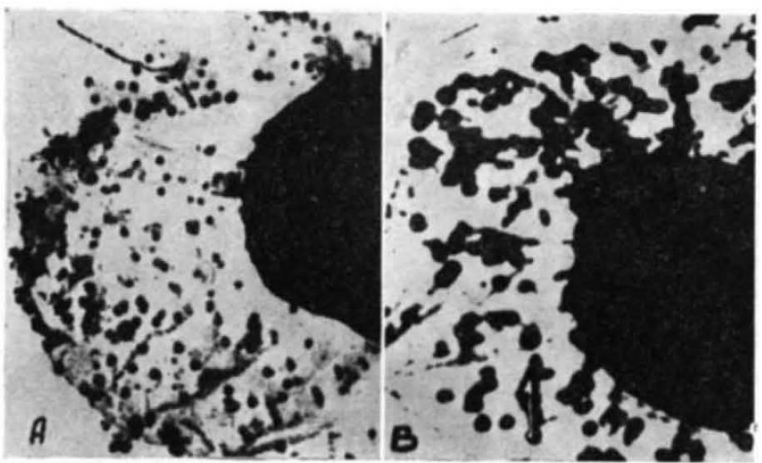

Fig. 1. Influenza virus $P R-8$ adsorbed on the chick red blood cell stroma, $A$, treated with $\mathrm{OsO}_{4} ; B$, treated with iodized immune $\gamma$-globulin

We have attempted, however, to estimate more precisely the possibility of obtaining a sufficient contrast by iodizing. For this purpose we used the photographs taken in the course of electron-microscopic examination of thin tissue sections of the murine thyroid ${ }^{11}$. Thyreoglobulin contains 0.8 per cent of iodin $\theta^{12}$. One could hope to detect it by electron-microscopic examination. Indeed, the photomicrographs showed several types of granules with high-density levels. Although there is no direct evidence that these granules are really the granules of thyreoglobulin, their unusual character, specificity for the thyroid gland and the characteristic dynamies of their occurrence suggested the possibility of obtaining a sufficient contrast by using iodized antibodies. On the basis of the aforementioned considerations the investiga tions described here were performed. Influenza virus (strain $P R-8$ ) was used as the antigen, and hyperimmune polyvalent horse serum as the source of antibodies.

(1) An allantoic culture of influenza virus (strain $P R-8)$ was purified by moans of ultrafiltration ${ }^{13}$ and differential centrifugation, as a result of which a virus suspension in 1 per cent ammonium acetate solution was obtained.

(2) $\gamma$-Globulin was prepared from hyperimmune horso serum ${ }^{14}$. 'The solution obtained was diluted with sodium bicarbonate solution, down to the $\gamma$-globulin concentration of 2 per cent and 1.5 per cent concentration of sodium bicarbonate. The solution was cooled down to $0^{\circ}$ to $+2^{\circ} \mathrm{C}$ in an ice bath with constant stirring by a magnetic stirrer and gradually was added dropwise $0.2 \mathrm{~N}$ iodine solution in othanol at a rate of about $0.01 \mathrm{ml} . / \mathrm{min}$. The amount of iodine added corresponded to 10 per cent of the weight of $\gamma$-globulin. The solution gradually became coloured and at the end of the iodine addition turned yellow, with a decrease of $p \mathrm{H}$ from $8 \cdot 3-8.4$ to $8 \cdot 2-8 \cdot \mathrm{I}$. Addition of iodine lasted $2-2 \cdot 5 \mathrm{~h}$. Then the solution was poured into a 'Cellophane' sac and dialysed overnight against 1 per cent ammonium acetate solution, $p \mathrm{H}$. 7.0. A colourless solution was centrifuged at $8,000-10,000$ r.p.m. for $20 \mathrm{~min}$. The residue was discarded and the solution was used as the source of iodized antibodies.

(3) The antigen-antibody reaction was performed in two ways: (i) a sodium periodate solution was added to the virus suspension. The non-reacted sodium periodate was reduced with glucose ${ }^{15}$. Then the virus-containing suspension was mixed with chick red blood cells stroma, adsorbing the virus on the stroma and adding the iodized antibodies according to the method described ${ }^{15}$; (ii) the virus suspension was mixed with antibodies, after which the mixture was spread over a colloid membrane.

(4) The initial non-iodized anti-influenza serum and the iodized horse anti-rabic serum were used as controls.

(5) The preparations were examined with an electron microscope, model $J E M-5 Y$, at an accelerating voltage of $80 \mathrm{kV}$. The expositions and the enlargements of the test and the control proparations were identical. Photographs of the materials were taken simultaneously at all stages.

Seligman ${ }^{16}$ pointed out that it is very desirable to introduce iodine into preparations examined with electron microscopes. When iodizing was performed, we tried to introduce an amount of iodine into the antibody molecule maximal from the point of view of preserving the specific activity. The first experiments showed sufficient stability of the iodized antibodies and the amount of iodine intro. duced was increased up to 5 por cont. This amount is more than twice as high as that calculated. In practice, 10 per cent of iodine is added to the $\gamma$-globulin solution, since only $\sim 50$ per cent of iodine added is incorporated into the $\gamma$-globulin molecule. Table I shows the results of detormining the antibody titres in the hæmagglutination inhibition reaction.

Table 1. Changes in the Titres of $\gamma$-Globllin Resultivg erox

\begin{tabular}{ccc}
$\begin{array}{c}\text { No. of } \\
\text { experi- }\end{array}$ & \multicolumn{2}{c}{ Titres of the hæmagglutination } \\
ments & \multicolumn{2}{c}{ inhibition reaction } \\
After iodizing \\
1 & $1: 5,120$ & $1 \vdots 1,024$ \\
2 & $1: 512$ & $1: 512$ \\
3 & $1: 512$ & $1: 128$
\end{tabular}

Howevor, since the analysis of the iodine content in the antibodies was not performed, it was difficult to say what was the real content of iodine in the antibodies. Still, the examination of the preparation with electron microscope demonstrated that, in both the first and the second

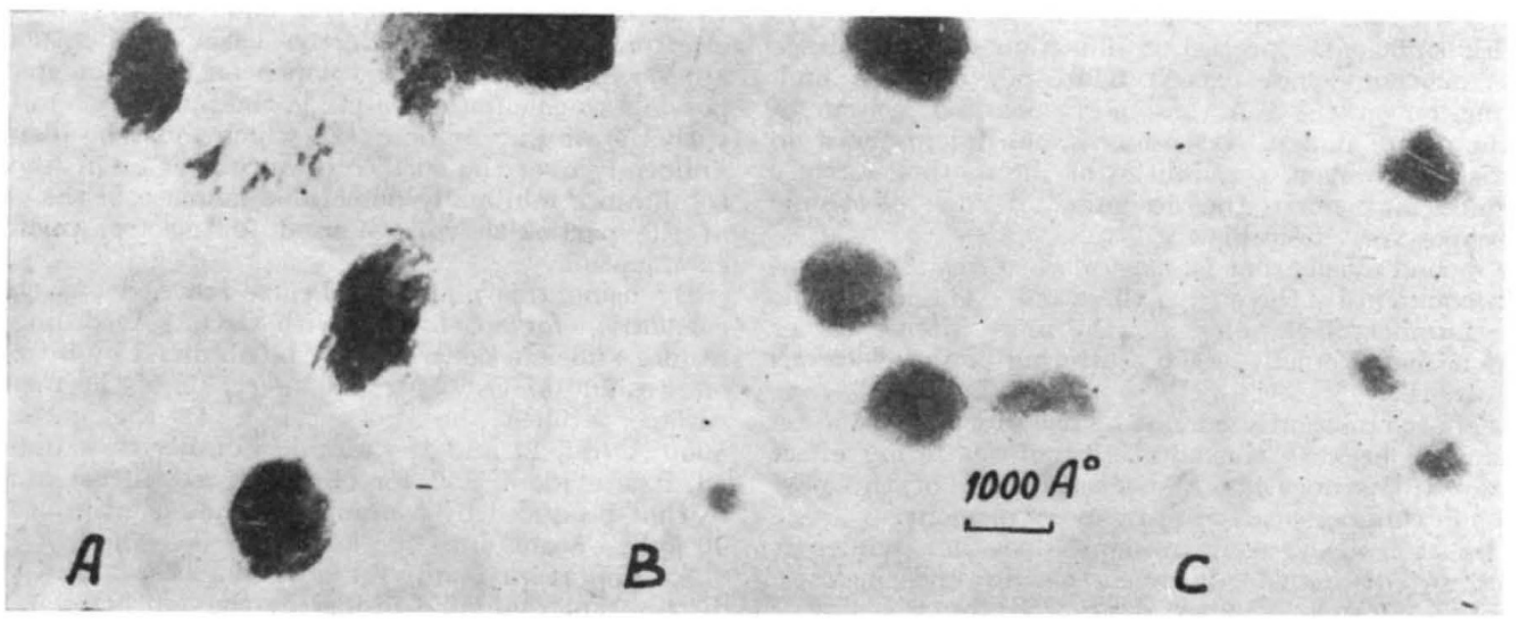

Fig. 2. Influenza virus $P R-8$ mixed with $\gamma$-globulin, $A$, with iodized immune $\gamma$-globulin; $B$, with non-iodized immune $\gamma$-globulin; $C$, with iodized anti-rabic $\gamma$-globulin $(\times 100,000)$ 
variations of the reaction virus-antibodies, the contrast is observed in the presence of sufficient specificity.

Fig. $1 A$ shows a preparation of influenza virus $P R-8$ adsorbed on the chick red blood cell stroma contrasted with $\mathrm{OsO}_{4}$ according to the usual procedure. Fig. $1 B$ shows an analogous preparation treated with iodized antibodies but not contrasted with $\mathrm{OsO}_{4}$. The complex virus-antibody is clearly seen on the red blood cell stroma. Fig. $2 A$ shows a preparation obtained on mixing a suspension of influenza virus $P R-8$ with iodized immune $\gamma$-globulin. A sufficient contrast of the complex virusantibody is clearly seen.

Fig. $2 B$ shows a preparation obtained in the same way by mixing a suspension of influenza virus $P R-8$ with noniodized immune $\gamma$-globulin. The complex virus-antibody is seen, as expected, in the form of a light grey diffuse zone.

Fig. $2 C$ shows a preparation, obtained with iodized antirabies horse $\gamma$-globulin instead of iodized anti-influenza $\gamma$-globulin. The virus particles are seen in the form of greyish diffuse spots.

The photomicrographs are demonstrating an undoubtedly sufficient level of contrast obtained with iodized antibodies. However, it should be remembered that the final conclusion as to whether iodized antibodies are applicable for cytological investigations with the use of electron microscopes may be drawn only on the basis of investigating ultra-thin tissue sections treated with similar antibodies.

'Pearse, E., Histochemistry (Russian ed.), 666 (Moscow, 1962).

${ }^{2}$ Singer, S., Nature, 183, 1523 (1959).

${ }^{3}$ Landsteiner, K., The Specificity of Serological Reactions (Harvard Univ. Press, Camb., Mass., 1945).

4 Sigel, M., Proc. Fifth Intern. Congr. Biochem. (Russian ed,), Sections 1-18 482 (Moscow, 1962).

${ }^{5}$ Kulberg, A. J., and Azadova, N. B., Vopr. virusol., No. 1, 100 (1963).

- Pepe, F., Finck, H., and Marshall, J., Anat. Rec., 136, 257 (1960).

${ }^{7}$ Pressman, D., and Sternberg, L. A., J. Amer. Chem. Soc., 72, 22 (1950).

${ }^{8}$ Cohen, S., J. Immunol., 67, 339 (1951).

- Zeitler, E., and Bohr, G. E., Exp. Cell. Res., 12, 44 (1957).

${ }^{10}$ Valentine, R. S., Nature, 181, 832 (1958).

${ }^{11}$ Ekholm, R., and Sjöstrand, F. S., J. Ultrastructur. Res., 1, 187 (1957).

12 Choh $\mathrm{Hao} \mathrm{Li}$, in The Proteins, 2, A, 595 (Acad. Press, N.Y., 1951).

1s Mekler, L. B., Frizuck, S. G., Naumova, V. K., Obukhova, V. R., and Demidova, S. A. (in the press).

14 Beilinson, A. V., Orlova, N. N., Shakhanina, K. L., Vitokhina, T. A. Chistoserdova, T. V., and Lvova, A. I., Vopr. virusol., No. 2, 140 (1960). is Powell, R. D., J. Lab. Clin. Med., 58, 386 (1961).

${ }^{16}$ Seligman, A. M., cited in ref. 1, p. 681 .

\title{
SORPTION OF WATER BY WOOL KERATIN
}

\author{
By J. G. DOWNES and P. NORDON \\ C.S.I.R.O. Division of Textile Physics, Wool Research Laboratories, Ryde, Sydney
}

$I^{N}$ a recent communication ${ }^{1}$, Dr. J. L. Morrison distinguishes a water-content range of $35-42$ per cent (relative to dry weight) in which he proposes that water is thermodynamically bound to wool keratin. His proposal is based on the results of experiments in which water vapour is slowly withdrawn via a capillary tube from a flask holding a wool sample initially containing free water, air boing absent. It is our opinion that water is not bound to keratin in the proposed range ( $B C$ in Morrison's notation) and that his obsorvations result from a failure to maintain the temperature throughout his sample sufficiently uniform. Our conclusions are based on theoretical grounds and are supported by results we have obtained from an experimental arrangement similar to that described by Morrison but used with a much reduced evacuation rate to obtain more nearly isothermal conditions.

The important role of temperature in the wool-water sorption system is not always fully appreciated. Two examples may serve to emphasize the magnitude of the effect: (1) if the water content of a wool sample near saturation is changed under adiabatic conditions by 1 per cent, then the temperature of the sample will change by about $12^{\circ} \mathrm{C}$. (2) In a system initially containing water vapour at saturation pressure at $20^{\circ} \mathrm{C}$, if the temperature is increased by $1^{\circ} \mathrm{C}$ while the vapour pressure is maintained constant, then the relative pressure (relative humidity) is reduced to 94 per cent and the corresponding equilibrium water content of wool is reduced from 33 to about 26 per cent.

Owing to the presence of these strongly coupled effects the mechanisms of heat transfer are of prime importance in experimental studies of sorption. It is not easy to make observations of changes in water content which are characteristic of the wool keratin alone and uninfluenced. by heat effects. This difficulty has already been considered by King and Cassie ${ }^{2}$. It is particularly apparent in vacuum systems where heat transfer by convection is not present.

In Morrison's experiment water is removed from the sample at an initial rato of $2 \cdot 2 \times 10^{-4} \mathrm{~g} / \mathrm{sec}$. Assuming the heat of evaporation as $580 \mathrm{cal} / \mathrm{g}$, then $0.128 \mathrm{cal} / \mathrm{sec}$ must be transferred to the sample. Transfer of heat to the interior of the wool mass is effective by conduction only at the vapour pressures used. Assuming the conductivity of the sample is effectively that of the water vapour present, for which we take the value $4.5 \times 10^{-5}$ $\mathrm{cal} / \mathrm{sec} / \mathrm{cm} /{ }^{\circ} \mathrm{C}$ (ref. 3) and assuming a spherical sample of diameter $5 \mathrm{~cm}$, it can be shown ${ }^{4}$ that the necessary heat transfer can only take place if the centre of the wool mass is about $45^{\circ} \mathrm{C}$ below the surface temperature. It is clear, therefore, that either the experiment is not isothermal or evaporation is not occurring uniformly through the sample.

If evaporation occurs only from the outer part of the mass a nuch smaller temperature difference is required to sustain the necessary heat flow. In any actual experiment the interior temperature of the mass will fall only slightly to correspond with the rate of evaporation from the interior, which is limited by the relatively slow rate at which heat can be conducted to it. During evacuation, therefore, the source of water is essentially only the outer region of the wool mass. In the range $A B$ (Morrison's notation) the free water content of the outer region becomes progressively reduced and is lower than the average for the whole sample; however, the vapour pressure is constant at the saturation value.

After point $B$ is passed it is our opinion that the water content of the outer part of the wool mass is being reduced during evacuation to values progressively lower than the saturation value (that is, that usually taken as 33-35 per cent for unmodified wool keratin ${ }^{5}$ ), while in the interior of the mass free water remains present but with its boundary receding towards the centre. The vapour pressure during evacuation will fall below the saturation value (this is manifested by the fall over the range $B C$ in the amount removed per 20-min evacuation). The vapour pressure of the free water inside the mass falls correspondingly because it is at the lower temperature necessary to maintain heat flow. However, in the range $B C$ the average moisture content for the whole sample is above the usually accepted saturation value for keratin ${ }^{5}$. 